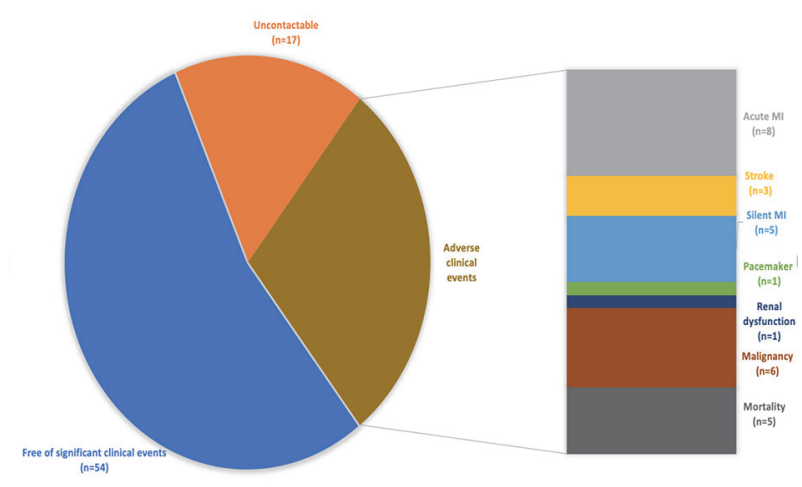

Abstract 19 Figure 2 Major adverse cardiovascular event rates The major adverse cardiovascular event rate ( $\mathrm{Ml}$, angina, revascularisation, CVA, death) during the 6-year follow-up period, including the patients with a silent $\mathrm{Ml}$, amounted to $25 \%$ in this study with an overall clinical event rate of $35 \%$

Results Follow-up was successful in 83 participants. Of those, 29 experienced cardiovascular/clinical events (36\%) (figure 2). Of the remaining 59, 32 patients who experienced no events received follow-up CMR. In this cohort, despite no significant changes in blood pressure, weight, or glycated-hemoglobin, significant reductions in biventricular end-diastolic-volumes and ejection fractions occurred over time (tables $1 \& 2$ ). The mean ECV was unchanged. Baseline plasma high-sensitivity cardiac-troponin-T (hs-cTnT) was significantly associated with change in left ventricular (LV) ejection fraction. Patients who experienced MACE had higher LV mass and greater LV concentricity than those who remained event-free.

Conclusions T2D results in reductions in biventricular size and systolic function over time even in the absence of cardiovascular/clinical events.

Conflict of Interest Nil

\section{THE COURSE OF MITRAL REGURGITATION DETECTED AFTER ACUTE MYOCARDIAL INFARCTION}

${ }^{1}$ Harish Sharma, ${ }^{2}$ Mengshi Yuan, ${ }^{1}$ Iqra Shakeel, ${ }^{3}$ Ashwin Radhakrishnan, ${ }^{1}$ Samuel Brown, ${ }^{2}$ John May, ${ }^{1}$ Nawal Zia, ${ }^{2}$ Kieran O'Connor, ${ }^{3}$ Sandeep Singh Hothi, ${ }^{4}$ Saul Myerson, ${ }^{2} \mathrm{M}$. Adnan Nadir, ${ }^{2}$ Richard P. Steeds. 'University of Birmingham, Birmingham, UK; ${ }^{2}$ Queen Elizabeth Hospital Birmingham; ${ }^{3}$ New Cross Hospital, Wolverhampton; ${ }^{4}$ Department of Cardiovascular Medicine, University of Oxford

\subsection{6/heartjnl-2021-BCS.20}

Background Mitral regurgitation (MR) is commonly observed following acute myocardial infarction (MI). Localised left ventricular (LV) remodelling in the region of papillary muscles together with impaired myocardial contractility promote MR. There is a paucity of long-term follow-up studies to determine whether the severity of MR observed post-MI, changes with time.

Purpose This study retrospectively followed up patients with MR detected following acute MI (AMI) to investigate changes in MR severity with time and assess for pre-discharge predictors of MR regression or progression.

Methods Clinical records of 1000 patients admitted with AMI between 2016 and 2017 to a single centre were retrospectively interrogated. One hundred and nine patients met the inclusion criteria of $\mathrm{MR}$ on pre-discharge transthoracic echocardiography (TTE) and follow-up TTE scans. Echocardiographic parameters were investigated to determine predictors of progression or regression at follow-up. Patients were divided according to those who had early follow-up TTE (within 1-year) and late follow-up TTE (beyond 1-year).

Results Early follow-up TTE was performed in 73 patients at a median of 6 (IQR 3-9) months. Patients had a mean age of $69 \pm 13$ years and were predominantly male 50/73 (68\%). At baseline, relative MR severities were: 49/73 (67\%) mild MR, 23/73 (32\%) moderate MR and 1 (1\%) severe MR. At follow-up, MR had completely resolved in 18/73 (23\%) patients, while 39/73 (53\%) had mild MR, 15/73 (21\%) moderate MR and 1 (1\%) severe MR (figure 1A). Compared to patients with no resolution of $\mathrm{MR}$, those with complete resolution were younger (mean age $62 \pm 16$ vs $72 \pm 11$ years; $\mathrm{p}=0.015$ ) but there were no other significant differences between the groups. Resolution at early follow-up did not significantly influence long-term mortality rates. Late followup TTE was performed in 69 patients at a median 2.4 (IQR 2-3.2) years. Pre-discharge, $49 / 69$ (71\%) patients had mild MR and 20/69 (29\%) moderate MR. At follow-up, MR had completely resolved in 18/69 (26\%), and amongst patients with persistent MR, proportion of severities were: 37/69 (54\%) mild MR, 11/69 (16\%) moderate MR and 3/69 (4\%) severe MR (figure 1B). Patients with progression of mild MR were more likely to have lower left ventricular ejection fraction (LVEF: $47 \pm 15$ vs $57 \pm 12 \% ; \quad \mathrm{p}=0.010$ ) and greater indexed left ventricular end-systolic volume (LVESVi: $37 \pm 23$ vs $25 \pm 14 \mathrm{ml} / \mathrm{m} 2 ; \mathrm{p}<0.001)$ on pre-discharge TTE. Resolution of MR at late follow-up was associated with a reduction
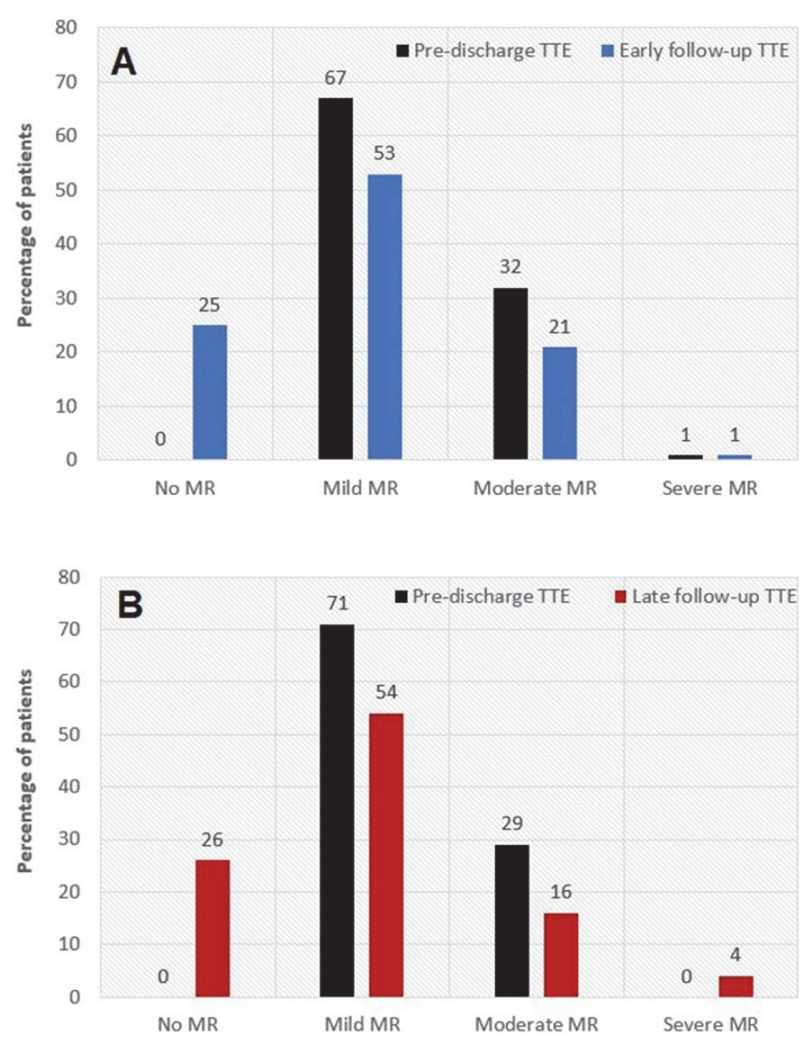

Abstract 20 Figure 1 (A) Progression of MR from pre-discharge TTE to early followinf-up TTE [median time 6(3-9) months]. (B) Progression of MR from pre-discharge TTE to late follow-up TTE [median time 2.4 (2.0-3.2) years] 
in long-term mortality [deaths: $2 / 55$ (3\%) vs 3/14 (21\%); $\mathrm{p}=0.022]$ at a mean follow-up of 4.2 years from $\mathrm{MI}$.

Conclusion MR observed following AMI resolved completely in approximately one-quarter of patients at 6-month and 2year follow-up. Progression of mild MR at long-term follow-up appears to be associated with increased mortality and is predicted by lower LVEF and greater LVESVi predischarge.

Conflict of Interest None

\section{LONG TERM FOLLOW-UP AND OUTCOMES AFTER VALVE REPLACEMENT - A 10-YEAR, SINGLE-CENTRE EXPERIENCE OF THE HEART VALVE SURVEILLANCE CLINIC}

${ }^{1}$ Polyvios Demetriades, ${ }^{2}$ Rosie Oatham, ${ }^{1}$ Cheryl Oxley, ${ }^{1} T i m$ Griffiths, ${ }^{1}$ Sally Clews, ${ }^{1}$ Nigel Stokes, ${ }^{1}$ Grant Heatlie, ${ }^{1}$ Simon Duckett. 'University Hospitals of North Midlands NHS Trust, Stoke-on-Trent, UK; ${ }^{2}$ School of Medicine, Keele University, Stoke-on-Trent, UK

\subsection{6/heartjnl-2021-BCS.21}

Introduction The long-term management of patients following valve replacement is challenging. The fields of percutaneous and surgical valves are expanding rapidly, leading to increased service demands. Most patients in our institution are managed within a dedicated cardiac physiologist' run valve clinic. Initially, follow-up centred around ESC 2012 guidelines on the management of valve disease, which recommended a baseline clinical and echocardiographic assessment after surgery and lifelong annual clinical follow-up. In addition, they recommended annual echocardiogram 5 years for bioprosthetic valves with no specific guidance for mechanical valves. Locally, all patients enrolled into the valve clinic received annual clinical and echocardiographic assessment. In 2019, the BHVS/ BSE published more comprehensive guidance on long-term follow-up of these patients. The Covid-19 pandemic placed pressure on the NHS to reduce outpatient appointments. Prior to service alteration, we conducted an audit to expand our understanding of outcomes in these patients.

Methods We retrospectively analysed the data of all patients enrolled in our valve service. We assessed demographics, date and indication for surgery, prosthesis type and position, baseline assessment, frequency of follow-up and significant valverelated complications. Complications constituted: any degree of paravalvular regurgitation, $\geq$ moderate transvalvular regurgitation, raised transvalvular gradients, valve thrombosis, infective endocarditis, new LV dysfunction, need for reintervention, cardiac-related hospital admission and valverelated death.

Results We identified 294 patients who underwent valve replacement since clinic establishment in 2010. Patient demographics are shown in table 1 . Only $37 \%$ of patients had baseline echocardiogram following surgery. Once enrolled into the clinic, $82.7 \%$ had yearly clinical and echocardiographic assessment. Table 2 demonstrates the echocardiographic and clinical complications we identified. During follow up $20.7 \%$ developed regurgitation, 9.5\% developed abnormal gradients and one required re-intervention for re-stenosis. One patient had valve thrombosis and was managed medically. Additionally, 9.2\% were diagnosed with new LV dysfunction; four of these required admission with decompensated heart failure and one died. 3.4\% developed infective endocarditis; three required redo surgery and four died. Figure 1 provides a schematic of valve-related complications and outcomes. Importantly, all patients who required admission, re-do surgery or that died, presented acutely with symptoms; the complications were not picked-up by the valve clinic.

Conclusions Contrast to our expectations, we identified only a small number of valve-related complications. With pressures rising to reduce outpatient footprint, we are now in the process of safely adjusting our practice in line with the BHVS/ BSE recommendations, supported by the evidence generated by our audit. We strongly encourage departments review their current services and implement evidence-based guidelines in

Significant valve-related complications

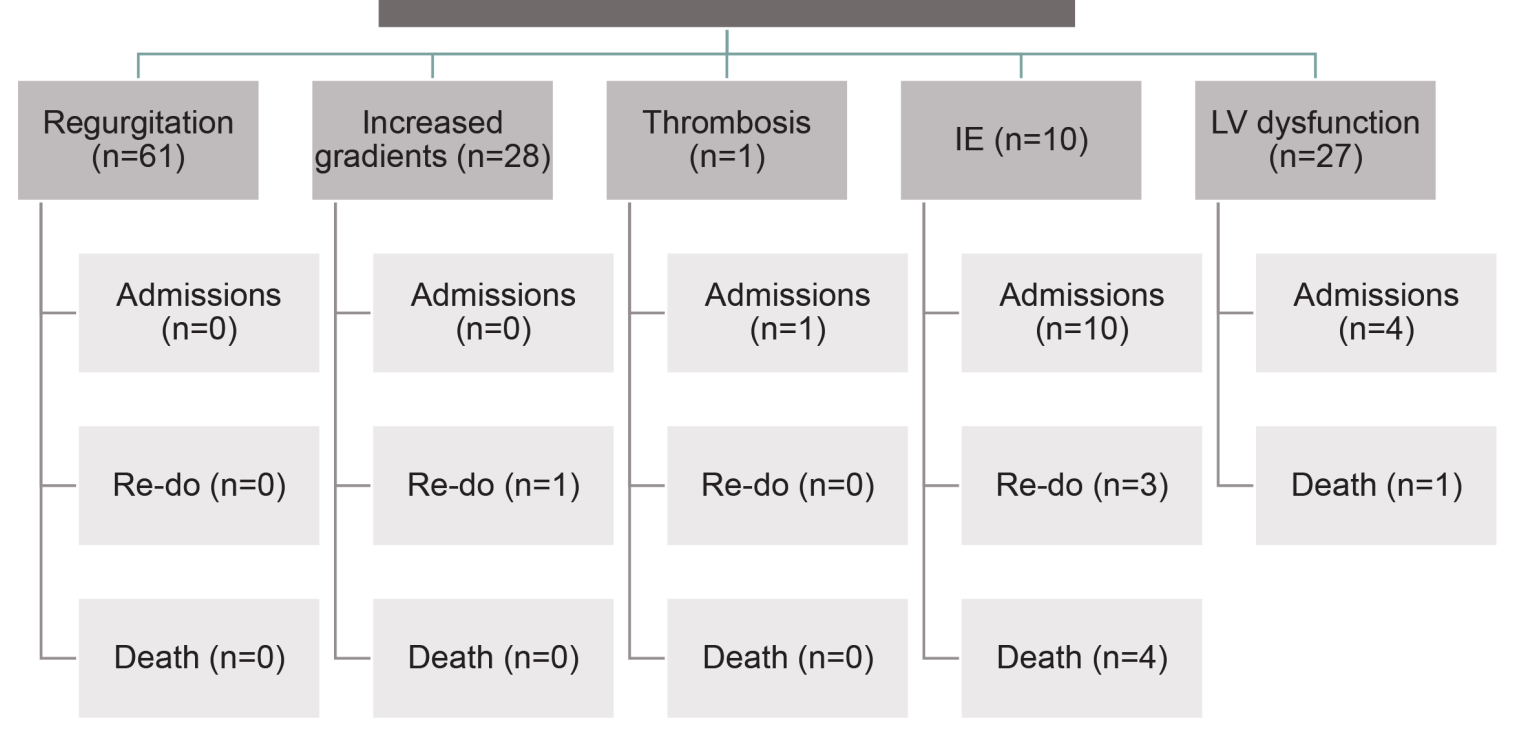

Abstract 21 Figure 1 Significant valve related complications and outcomes

${ }^{*}$ Regurgitation = any degree of paravalvular / $\geq$ moderate transvalvular; Increased gradients = above normal for specific valve type; IE=infective endocarditis 